\title{
Inséminations intra-utérines avec sperme du conjoint dans les indications masculines : influence de l'âge de l'homme
}

\author{
C. MATHIEU*, R. ECOCHARD**, V. BIED*, J. LORNAGE*, J.C. CZYBA* \\ * Laboratoire de Biologie de la Reproduction; Hôpital Edouard Herriot ; Lyon \\ ** Centre d'Information Médicale ; Hôtel-Dieu. 1 ; Lyon
}

\section{RESUME}

Nous avons analysé les résultats de $\mathbf{4 9 2}$ cycles d'Insémination Artificielle avec sperme du Conjoint (IAC) par voie Intra-Utérine (IU) chez 152 couples en utilisant la méthode des courbes de survie en modèle de Cox. 36 grossesses ont été obtenues. Le taux cumulé de grossesse était de $20 \%$ à 3 cycles et de $31 \%$ à 6 cycles. L'analyse univariée n'a pas révélé de facteur pronostique de succès. L'analyse multivariée a fait apparaître que l'âge de l'homme $\geq 35$ ans était un facteur de mauvais pronostic $(p=0,04)$ de même que l'existence d'une dysovulation. L'âge de la femme $>35$ ans contrairement à notre attente n'est pas apparu comme un facteur de pronostic statistiquement significatif après prise en compte des autres facteurs.

Mots clés : Insémination Artificielle, Infertilité masculine, oligoasthénotératozoos permie, courbes de survie, modèle de Cox.

\section{INTRODUCTION}

L'insémination intra-utérine avec sperme du conjoint (IAC IU) dans les indications masculines est de pratique répandue mais fait l'objet de publications contradictoires. Pour certains auteurs $[5,7,12,15,17,18$, 29] les résultats sont satisfaisants alors que pour d'autres les taux de succès sont faibles voire nuls $[9,14,26]$. Nous rapportons ici notre expérience de l'IAC IU dans ces indications et plus particulièrement en cas d'anomalies non spécifiques du sperme (sont exclues les éjaculations rétrogrades et les auto-immunisations antispermatozoïdes). Nous nous sommes attachés à analyser les résultats en méthode actuarielle, méthode recommandée par SchWARTz [22] car elle prend en compte la durée de l'exposition au traitement pour chaque couple sans attribuer un "poids" excessif à chaque couple de la série. Dans cette étude nous avons cherché à dégager les facteurs pronostiques du bilan d'infertilité des couples.

\section{MATERIEL ET METHODES}

\section{Patients}

Cette étude regroupe 152 couples traités dans le service de Biologie de la Reproduction (Hôpital E.Herriot - Lyon) ayant commencé les IAC à partir du 1.09.87. L'observation s'arrête au 31.12.92 date à laquelle les patients n'ont pas tous achevé leur traitement (492 cycles). Les données concernant ces couples ont été enregistrées sur un fichier informatisé déclaré à la Commission Nationale Informatique et Liberté (CNIL). La base de données a été installée sur DBASE IV (Ashton Tate Corporation -Version Française : la Commande Electronique). Chaque dossier est divisé en 2 fichiers : 1 fichier fixe où figurent les données du bilan d'infertilité et un fichier "cycles" où sont enregistrées les séquences de traitement et leur résultat. 
Les patientes ont eu un bilan comprenant :

- courbe de température et bilan hormonal

- hystéro-salpingographie

- test post-coïtal et/ou test de pénétration croisé de la glaire cervicale

- sérologies de la rubéole, de la toxoplasmose, des chlamydiae et de l'infection à virus de l'immuno déficience humaine (VIH).

Les hommes ont eu un bilan comprenant:

- spermogramme-spermocytogrammespermoculture- MAR test

- un test de sélection des spermatozoïdes afin de s'assurer qu'il était possible d'inséminer au moins 300000 spermatozoïdes mobiles à morphologie normale,

- sérologies des chlamydiae et de l'infection à VIH.

\section{Préparation du sperme}

Toutes les inséminations ont été réalisées avec des spermatozoïdes sélectionnés après traitement du sperme in vitro. La préparation du sperme a été soit la migration ascendante (45 cycles), soit la centrifugation sur gradient de Percoll [13, 20] à 2 densités (40 et $70 \%$ Percoll) ou 3 densités $(40,60$ et $80 \%$ Percoll) (447 cycles).

Quelle que fût la méthode utilisée, les spermatozoïdes étaient remis en suspension dans $0,5 \mathrm{ml}$ de milieu $\mathrm{B} 2$ et un volume de $0,3 \mathrm{à} 0,4 \mathrm{ml}$ était utilisé pour l'insémination. Le nombre de spermatozoïdes mobiles utilisés pour l'IAC n'excédait pas $10 \mathrm{mil}$ lions.

\section{Stimulation de l'ovulation}

En première intention, nous avons utilisé le citrate de clomifène (CC) sauf en cas d'échec de stimulation reconnu antérieurement avec ce produit ou en cas de contre-indication à celui-ci ( 224 cycles). Le protocole comprenait :

- la prise de 50 ou 100 mg de CC (Pergotime ${ }^{\circledR}$ Serono, France) du 3ème au 7ème jour du cycle.
- la surveillance de l'ovulation par monitorage échographique à partir du 11ème jour du cycle.

Nous avons utilisé la stimulation de l'ovulation par $\mathrm{hMG}$ en deuxième intention après échec de 2 ou 3 cycles d'IAC sous CC ou en première intention lorsqu'il n'était pas possible d'utiliser le CC (160 cycles). Le protocole comprenait :

- l'injection intra-musculaire de $150 \mathrm{UI}$ d'hMG (Néo-Pergonal ${ }^{\circledR}$ Serono, France ou Humegon ${ }^{\circledR}$ Organon, France ou Inductor ${ }^{\circledR}$ Pharmagyne, France) aux 4ème, 6ème 8ème 9 ème jours du cycle.

- la surveillance par un dosage plasmatique estradiol et un monitorage échographique de la maturation folliculaire de manière à adapter la posologie jusqu'à obtention d'un follicule mûr et d'un taux satisfaisant d'estradiol.

Dans les mêmes circonstances que pour la stimulation par hMG, nous avons utilisé la stimulation par FSH (39 cycles). Le protocole comprenait :

- l'injection intra-musculaire de 75 UI de FSH (Métrodine ${ }^{\circledR}$ Serono, France ou Fertiline ${ }^{\circledR}$ Pharmagyne, France) du 4ème au 9ème jour du cycle.

- une surveillance de l'ovulation comparable à celle du protocole hMG.

Au cours de 36 cycles, l'ovulation n'a pas été stimulée et 13 cycles ont été réalisés sous des protocoles divers tels que CC-hMG ou FSH-hMG.

\section{Déclenchement de l'ovulation}

L'ovulation a été déclenchée à maturité folliculaire (follicule $\geq 16 \mathrm{~mm}$ de diamètre et dosage d'estradiol $\geq 300 \mathrm{pg} / \mathrm{ml}$ dans les cas où l'estradiol était dosé) par l'injection intra-musculaire de 5000 UI d'hCG (hCG Endo ${ }^{\circledR} 5000$ unités-Organon, France). Deux IAC IU ont été pratiquées 16 et 40 heures après le déclenchement de l'ovulation (334 cycles) ; une seule IAC a été réalisée en cas de décalage net de la température après la première IAC. 
L'ovulation n'a pas été déclenchée et le cycle d'IAC a été annulé s'il existait plus de 3 follicules de diamètre $\geq 14-15 \mathrm{~mm}$ au moment du déclenchement et/ou si le taux d'estradiol plasmatique dépassait $2000 \mathrm{pg} / \mathrm{ml}$. Ces mesures avaient pour but de réduire le risque de grossesse multiple et/ou d'hyperstimulation ovarienne grave.

\section{Soutien de la phase lutéale}

Après stimulation par $\mathrm{CC}$, le traitement de la phase lutéale a consisté en l'injection intra-musculaire de 1500 UI d'hCG (hCG Endo ${ }^{\circledR} 1500$ unités) 4 et 8 jours après le déclenchement de l'ovulation par hCG 5000 UI.

En cas de stimulation par hMG ou par FSH, diverses situations pouvaient se présenter :

- pas de traitement de la phase lutéale

- soutien par hCG dans les mêmes conditions que pour la stimulation par CC

- soutien par progestérone seule: Utrogestan $^{\circledR}$ (Besins-Iscovesco, France) 3 comprimés par jour, ou Duphaston ${ }^{\circledR}$ (Duphar, France) 2 comprimés par jour.

- soutien par association hCG-progestérone.

Le choix de ces traitements se faisait en fonction des données du cycle en cours et/ou des cycles précédents lorsque ceux-ci montraient des phases lutéales courtes.

\section{Réalisation de 1'IAC}

Les IAC ont été réalisées à l'aide d'une petite sonde (Sondjet $\mathrm{S}_{10} \mathrm{CH}_{8}$ - Laboratoires Peters-Aubervilliers, France ou sonde intra-utérine de Kremer- DelafontaineLaboratoire CCD, Paris, France).

\section{Nombre de cycles de traitement}

Six cycles d'IAC IU ont été proposés aux couples.

\section{Analyse statistique}

Pour procéder à l'analyse des résultats, il est nécessaire d'effectuer une fusion des fichiers "fixe" (bilan de stérilité) et "cycles".
Les taux de grossesse ont été calculés selon la méthode actuarielle en prenant en compte seulement la première grossesse obtenue par un couple. Il s'agit d'une méthode d'analyse comparable à celle des "courbes de survie".

L'analyse des facteurs influençant l'obtention ou non d'une grossesse a été faite en modèle univarié ayant recours au test du Log Rank puis en modèle multivarié. Le modèle multivarié utilisé est un modèle de Cox sur données discrètes (unité de temps=cycle) avec adaptation logistique du modèle de Cox $[6,16,28]$. La procédure utilisée pour la sélection des variables entrées dans le modèle est de type "stepwise". Les variables sont testées progressivement. Celles-ci sont choisies dans l'ordre suivant :

- la première est la plus significative en univariée

- puis vient la meilleure variable sur le plan de la significativité après prise en compte de la ou des précédentes.

Ces analyses ont été faites grâce au logiciel SAS avec les procédures SAS/STAT ${ }^{\circledR}$ et SAS PHREG $^{\circledR}$ (SAS Institute Inc.Cary NC USA).

\section{RESULTATS}

L'analyse des résultats a porté en méthode actuarielle sur 36 grossesses survenues au cours de 492 cycles. Le taux de faussescouches a été de 19,4\% (7/36). Les caractéristiques générales des couples étaient les suivantes:

- Durée de l'infertilité :

$$
4,1 \text { ans } \pm 3,1
$$

- Age de la femme :

31,41 ans $\pm 4,8$

- Age de l'homme : 33,9 ans $\pm 5,8$

- Infertilité primaire chez la femme : $66,4 \%$ des cas

- Infertilité primaire chez l'homme : $66,4 \%$ des cas

- Indication masculine : $\mathrm{N}=70$

- Indication mixte (cause féminine associée) : $\mathrm{N}=82$ 
Les données du bilan d'infertilité chez les femmes et les hommes sont résumées sur les tableaux 1 et 2 .

Les infertilités pour lesquelles un facteur masculin avait été retenu alors que les données du spermogramme étaient normales $(N=12)$ concernaient des patients présentant un défaut de pénétration du mucus cervical et/ou des perturbations des paramètres du mouvement vérifiés à plusieurs reprises.

Nous avons étudié l'influence de différents facteurs suivant un modèle univarié puis un modèle bivarié et enfin nous avons réalisé une analyse multivariée. Nous avons pu vérifier que la population restait homogène au cours de ces cycles en ce qui concernait les éléments du bilan d'infertilité (âge des patients, durée de l'infertilité, anomalies du spermogramme, ....). Par contre, le mode de stimulation de l'ovulation variait au cours des cycles : $59 \%$ de stimulation par citrate de clomifêne au premier cycle contre $18 \%$ au sixième cycle.

\section{ANALYSE UNIVARIEE}

Le taux de grossesse en fonction du rang du cycle est indiqué sur le tableau 3. La probabilité de conception après 3 cycles d'IAC est de $20 \%$ et atteint $31 \%$ après 5 cycles. Nous avons observé un faible taux de succès au 3 ème cycle $(3,4 \%)$ et nous n'avons pas obtenu de grossesse au 6ème cycle.

A partir de 35 ans, la probabilité de conception chute chez l'homme et chez la femme (Tableau 4) mais la différence n'est pas statistiquement significative par rapport aux autres classes d'âge.

L'existence d'une dysovulation (Tableau 5) diminue les chances de grossesse mais la différence n'est pas statistiquement significative. L'anovulation, de meilleur pronostic, ne concernait que 14 patientes.

Tableau 1 : Données du bilan d'infertilité chez les femmes.

\begin{tabular}{llc}
\hline & Nombre de patientes & $\%$ \\
\hline Trompes normales & $\mathrm{N}=131$ & $(82,2)$ \\
Cavité utérine normale & $\mathrm{N}=138$ & $(90,8)$ \\
Endométriose & $\mathrm{N}=5$ & $(3,3)$ \\
Ovulation normale & $\mathrm{N}=42$ & $(27,6)$ \\
Dysovulation & $\mathrm{N}=96$ & $(63,2)$ \\
Anovulation & $\mathrm{N}=14$ & $(9,2)$ \\
Hyperprolactinémie & $\mathrm{N}=16$ & $(10,5)$ \\
Test post-coïtal anormal & $\mathrm{N}=132$ & $(86,8)$ \\
\hline
\end{tabular}

Tableau 2 : Données du bilan d'infertilité chez les hommes:

Nombre de patients

$\%$

Spermogramme normal

$N=12$

$(7,9)$

Anomalies non spécifiques du sperme

Oligozoospermie

$\mathrm{N}=14$

$(9,2)$

Asthénozoospermie

$\mathrm{N}=30$

$(19,7)$

Tératozoospermie

$\mathrm{N}=2$

$(1,3)$

Oligo-asthénozoospermie

$\mathrm{N}=42$

$(27,6)$

Oligo-tératozoospermie

$\mathrm{N}=4$

$(2,6)$

Asthéno-tératozoospermie

$\mathrm{N}=20$

$(13,2)$

Oligo-asthéno-térato-zoospermie

$\mathrm{N}=24$

$(15,8)$

Polyzoospermie

$\mathrm{N}=4$

$(2,6)$ 
Tableau 3 : Taux de grossesse par cycle et cumulé selon la méthode actuarielle dans les indications masculines et mixtes des IAC IU - 152 couples.

\begin{tabular}{lllllll}
\hline Cycle & $\begin{array}{l}\text { Patients } \\
\mathbf{N}=\end{array}$ & $\begin{array}{l}\text { Grossesses } \\
\mathbf{N}=\end{array}$ & $\begin{array}{l}\text { Abandons } \\
\mathbf{N}=\end{array}$ & $\begin{array}{l}\text { Taux de grossesse/Taux cumulé de grossesse } \\
\text { cycle } \%\end{array}$ & $\begin{array}{l}\text { \% } \\
\text { Intervalle } \\
\text { de confiance } \\
\text { à 95\% }\end{array}$ \\
\hline 1 & 152 & 11 & 17 & 7,24 & 7,24 & $3,12-11,36$ \\
2 & 124 & 14 & 24 & 11,29 & 17,63 & $\mathbf{1 1 , 3 3 - 2 3 , 9 3}$ \\
3 & 89 & 3 & 26 & 3,37 & 20,4 & $\mathbf{1 3 , 5 8 - 2 7 , 2 3}$ \\
4 & 60 & 4 & 14 & 6,67 & 25,71 & $\mathbf{1 7 , 6 0 - 3 3 , 8 2}$ \\
5 & 42 & 3 & 17 & 7,14 & 30,89 & $21,46-40,32$ \\
6 & 22 & 0 & & - & - & - \\
\hline
\end{tabular}

Tableau 4: Influence de l'âge de la femme et de l'homme.

\begin{tabular}{|c|c|c|c|c|c|}
\hline & \multicolumn{3}{|c|}{$\begin{array}{l}\text { Taux cumulé de grossesse à } 5 \text { cycles \% } \\
\text { (intervalle de confiance à } 95 \%)\end{array}$} & \multirow[t]{2}{*}{$\begin{array}{c}\text { Log } \\
\text { Rank }\end{array}$} & \multirow[t]{2}{*}{$\mathbf{p}$} \\
\hline & $<30$ ans & 30-34 ans & $\geq 35$ ans & & \\
\hline Age de la femme & $\begin{array}{c}29,99 \\
{[15,13-44,85}\end{array}$ & $\begin{array}{c}33,52 \\
{[18,10-48,98]}\end{array}$ & $\begin{array}{c}24,53 \\
{[7,47-41,59]}\end{array}$ & 0,58 & 0,75 \\
\hline Age de l'homme & $\begin{array}{c}33,55 \\
{[9,77-57,32]}\end{array}$ & $\begin{array}{c}41,12 \\
{[24,74-57,50]}\end{array}$ & $\begin{array}{c}19,79 \\
{[7,38-32,21]}\end{array}$ & 4,50 & 0,11 \\
\hline
\end{tabular}

L'analyse de l'influence de différents facteurs présentée sur le tableau 6 ne montre pas de différence statistiquement significative pour ce qui concerne la durée de l'infertilité, l'association à un facteur féminin d'infertilite (indication mixte), le caractère primaire ou secondaire de l'infertilité, la présence d'une asthénozoospermie ou d'une oligozoospermie.

\section{ANALYSE BIVARIEE}

En analyse univariée, aucun facteur ne parait avoir de valeur pronostique statistiquement significative. La valeur la plus basse de $p$ est 0,11 pour l'âge de l'homme. Afin d'éliminer l'influence de facteurs confondants tels que l'âge de la femme, l'existence d'une dysovulation, la durée de l'infertilité ou la qualité du sperme, nous avons réalisé une analyse bivariée associant l'âge de l'homme à ces différents facteurs, tableau 7 ; l'influence de l'âge de l'homme se confirme puisque les valeurs de p sont inférieures à $0,11(0,10$ à 0,5$)$.

A l'inverse, l'âge de la femme après prise en compte de l'âge de l'homme, n'a pas de valeur pronostique statistiquement significative ( $\log \operatorname{Rank} 0,76 \mathrm{p}=0,38)$.

\section{ANALYSE MULTIVARIEE}

L'analyse multivariée (Tableau 8) fait apparaître l'âge de l'homme $\geq 35$ ans comme le premier facteur pronostique statistiquement significatif $(p=0.04)$. Puis sont apparues successivement: la dysovulation, l'oligozoospermie (comprenant l'oligozoospermie seule ou associée à d'autres anomalies du sperme), l'infertilié secondaire chez la femme et enfin l'infertilité secondaire chez l'homme.

L'expression du risque relatif correspondant à chacune de ces variables fait ressortir que l'âge de l'homme $\geq 35$ ans, l'existence d'une 
Tableau 5 : Influence de la qualité de l'ovulation.

Taux cumulé de grossesse à 5 cycles \% (intervalle de confiance à 95\%)

\begin{tabular}{ccccc}
\hline Anovulation & Dysovulation & Normale & Log Rank & p \\
\hline 70,15 & 24,14 & 33,91 & 4,16 & 0,13 \\
{$[26,49-1]$} & {$[13,91-34,37]$} & {$[15,31-52,51]$} & & \\
\hline
\end{tabular}

Tableau 6 : Analyse univariée de l'influence de différents facteurs pronostiques chez 152 couples.

\begin{tabular}{lcccc}
\hline & \multicolumn{2}{c}{ Taux cumulé de grossesse à 5 cycles } \\
\% (intervalle de confiance à 95\%) & non & $\begin{array}{c}\text { Log } \\
\text { Rank }\end{array}$ & p \\
\cline { 2 - 5 } Variable & oui & 26,86 & 1,32 & 0,25 \\
\hline Indication mixte & 34,09 & $(13,18-40,54)$ & & \\
Infertilité primaire & $(21,07-47,11)$ & 31,33 & 0,37 & 0,55 \\
pour la femme & $(18,83-42,81)$ & $(16,54-46,13)$ & & \\
Infertilité primaire & 35,55 & 20,99 & 0,89 & 0,35 \\
pour l'homme & $(23,21-47,89)$ & $(8,58-33,40)$ & & \\
Asthénozoospermie & 25,99 & 44,32 & 1,41 & 0,24 \\
Oligozoospermie & $(15,99-35,40)$ & $(23,47-65,17)$ & & \\
& 37,98 & 22,58 & 2,28 & 0,13 \\
Durée de l'infertilité & $(24,57-51,39)$ & $(9,93-35,23)$ & & \\
$>$ 3 ans & 27,78 & 38,60 & 1,44 & 0,23 \\
\hline
\end{tabular}

dysovulation chez la femme et le caractère secondaire de l'infertilité chez l'homme sont des facteurs de mauvais pronostic. Par contre la présence d'une oligozoospermie et le caractère secondaire de l'infertilité chez la femme sont des facteurs de bon pronostic. La valeur de p dans le modèle 5 pour l'oligozoospermie est 0,076 , c'est pourquoi en l'absence de valeur statistiquement significative, l'intervalle de confiance du risque relatif n'est pas exprimé. Le modèle 5 reste toutefois globalement significatif.

\section{DISCUSSION}

L'intérêt de l'IAC IU dans les indications masculines est encore débattu et une partie des divergences d'appréciations réside peut- être dans le fait que les indications masculines sont très hétérogènes, la sévérité de l'atteinte spermatique étant variable d'une étude à l'autre. D'autre part les comparaisons entre les études sont limitées en raison des différences d'expression des résultats. Le taux cumulé de grossesse en méthode actuarielle permet cette comparaison mais peu d'études l'utilisent. Nous avons obtenu un taux cumulé de grossesse à 5 cycles de $31 \%$; LALICH et coll. [19] remettent en question l'intérêt de l'IAC IU dans les indications masculines car le taux cumulé de grossesse à 6 cycles n'est que de 16,7\% dans leur expérience. BARRI et coll. [3] ainsi que FRIEIMAN et coll. [10] rapportent des taux cumulés de $16 \%$ et $38,3 \%$ respectivement mais dans une étude concernant 
Tableau 7 : Analyse bivariée. Valeur pronostique de l'áge de l'homme après prise en compte de l'age de la femme, de l'existence d'une dysovulation, de la durée de l'infertilité ou de la qualité du sperme. 152 couples - 492 cycles - 36 grossesses.

\begin{tabular}{lll}
\hline 2ème Variable prise en compte & Log Rank & p \\
\hline Age de la femme & 3,69 & 0,06 \\
Dysovulation & 4,01 & 0,05 \\
Durée de l'infertilité & 3,42 & 0,07 \\
Asthéno et/ou tératozoospermie & 2,76 & 0,10 \\
\hline
\end{tabular}

Tableau 8: Analyse multivariée de différents facteurs pronostiques 152 couples - 492 cycles36 grossesses.

Modèle de Cox

\begin{tabular}{llll}
\hline Modèle & \multicolumn{1}{c}{ Variables } & Valeur du test & p \\
\hline 1 & âge de l'homme $\geq 35$ ans & 4,05 & 0,04 \\
2 & $\begin{array}{l}\text { âge de l'homme } \geq 35 \text { ans -Dysovulation } \\
\text { âge de l'homme } \geq 35 \text { ans -Dysovulation }\end{array}$ & 7,66 & 0,02 \\
& $\begin{array}{l}\text { Oligozoospermie } \\
\text { âge de l'homme } \geq 35 \text { ans-Dysovulation } \\
\text { oligozoospermie- infertilité secondaire } \\
\text { chez la femme }\end{array}$ & 10,81 & 0,01 \\
& $\begin{array}{l}\text { âge de l'homme } \geq 35 \text { ans- Dysovulation } \\
\text { oligozoospermie -Infertilité secondaire } \\
\text { chez la femme-Infertilité secondaire chez }\end{array}$ & 13,55 & 0,008 \\
l'homme & & 0,002 \\
& & & \\
\hline
\end{tabular}

Risque relatif correspondant à chacune de ces variables après prise en compte des autres

\begin{tabular}{lll}
\hline & Risque relatif & intervalle de confiance à 95\% \\
\hline Age de l'homme $\geq 35$ ans & 0,41 & $0,18-0,97$ \\
Dysovulation & 0,45 & $0,22-0,93$ \\
Oligozoospermie & 1,88 & \\
Infertilité secondaire chez la femme & 5,73 & $2-16$ \\
Infertilité secondaire chez l'homme & 0,23 & $0,07-0,75$ \\
\hline
\end{tabular}

toutes les indications des IAC. L'expression des résultats en méthode actuarielle permet également de comparer l'efficacité de 2 traitements tels que les IAC ou Fécondation In Vitro (FIV). TAN et coll. [25] obtiennent $28 \%$ de succès après 3 cycles de FIV (20,4\% en IAC dans notre étude), TrouNsoN et coll. [30] font état d'un taux cumulé de grossesse en FIV de $20 \%$ après 2 cycles (17,6\% en IAC dans notre étude). Bien qu'il s'agisse dans ces 2 études d'indications masculines, des réserves doivent être faites en ce qui concerne les différences possibles entre les patients en FIV et ceux en IAC, les premiers pouvant présenter des caractéristiques de sperme ne permettant pas le recours aux IAC. Néanmoins ces réserves étant faites, la pratique de l'IAC IU apparait raisonnable en terme de succès dans les indications masculines; de plus il s'agit d'une technique moins invasive pour les femmes que ne l'est la FIV. 
L'analyse de nos résultats fait apparaître l'âge de l'homme comme le premier facteur de pronostic. Paradoxalement, l'âge de la femme n'apparait pas comme un facteur négatif de pronostic. Ceci va à l'encontre d'autres études [3, 21, 24]. Nous n'avons pas d'explication à cette constatation; nous pouvons formuler deux hypothèses :

1. les patientes âgées de 35 ans et plus ont reçu des séquences thérapeutiques différentes de celle des patientes plus jeunes ; le traitement des patientes de plus de 35 ans serait plus efficace et atténuerait l'effet négatif de l'âge habituellement observés.

2. la dysovulation est un facteur de pronostic péjoratif (le 2ème dans notre analyse multivariée) et la dysovulation (présente chez $63 \%$ des patientes de notre série) serait de mauvais pronostic quel que soit l'âge de la femme.

Pour vérifier la première hypothèse, nous allons procéder à l'analyse des facteurs variant à chaque cycle (stimulation de l'ovulation, qualité du sperme) ; les méthodes statistiques complexes qui doivent être mises en œuvre sont en cours de développement.

La mise en évidence du rôle de l'âge de l'homme nous a étonnés ; cependant, l'influence de l'âge de l'homme sur la fertilité est retrouvée dans plusieurs études $[1,4$, $8,11,23]$. L'âge paternel pourrait en outre jouer un rôle dans la qualité du conceptus $[2,27]$. De façon surprenante, chez l'homme le caractère secondaire de l'infertilité est également apparu comme de mauvais pronostic ; il n'est pas exclu que ces patients aient subi une altération suffisamment importante des caractéristiques de leur sperme au point d'être amenés à consulter pour infertilité. D'autre part, les chances de procréation pour ces hommes sont identiques à celles des hommes présentant une infertilité primaire au cours des 3 premiers cycles d'IAC (données non montrées) puis le taux de succès s'effondre après le 3ème cycle. Il y a là encore une probable sélection dans le temps d'hommes particulièrement hypofertiles.

\section{CONCLUSION}

L'IAC intra-utérine nous apparait licite en tant que procréation médicalement assistée de première intention dans les indications masculines. Nous avons étudié la valeur pronostique des éléments fixes du bilan d'infertilité des couples. L'accroissement de l'âge de l'homme est le paramètre de plus mauvais pronostic. Ce rôle de l'âge de l'homme a été retrouvé dans d'autres études où les résultats étaient analysés comme dans notre série selon la méthode des courbes de survie en modèle de Cox. L'analyse des résultats en prenant en compte les facteurs variant à chaque cycle permettra de préciser la valeur pronostique de la qualité du sperme ainsi que la supériorité ou l'équivalence des différents traitements de stimulation de l'ovulation.

\section{REFERENCES}

1. ANDERSON B. : Male age and fertility. Results from Ireland prior to 1911. Pop. Index., 1975, 41, 561-566.

2. AUROUX M. : Age du père et développement. Contracept. Fertil. Sex., 1993, 21, 382-385.

3. BARRI P.N., BUXADERAS, R., COROLEU, B., GENOVER, O., PARDO, M. AND CALDERON, G. : Résultats de l'insémination artificielle conjugale (IAC) après Percoll. Rev. Fr. Gynecol. Obstet., $1988,83,587-591$.

4. BOSTOFTE E., BAGGER P., MICHAEL A., STAKEMANN G. : Fertility prognosis for infertile men: results of follow-up study of semen analysis in infertile men from two differente populations evaluated by the Cox regression model. Fertil. Steril., 1990, $54: 1100-1106$.

5. BYRD N., ACKERMAN G.E., CARR B.R., EDMAN, C.D., GUZICK, D.S. AND MC CONNELL, J.D. : Treatment of refractory infertility by transcervical intra-uterine insemination of washed spermatozoa. Fertil. Steril., 1987, 48, 921-927.

6. COX D.R. : Regression model and life tables. Journal of the Royal Statistical Society Series B., 1972, $34,187-220$. 
7. CRUZ R.I., KEMMANN E., BRANDEIS V.T., BECKER K.A., BECK M., BEARDSLEY L. AND SHELDEN R. : A prospective study of intrauterine insemination of processed sperm from men with oligoasthenospermia in superovulated women. Fertil. Steril., 1986, 46, 673-677.

8. DUCOT B., SPIRA A., FENEUX D. AND JOUANNET P. : Male factors and the likelihood of pregnancy in infertility couples. II Study of clinical characteristics - pratical consequences. Int. J. Androl., 1988, 11, 395-404.

9. EMPERAIRE J.C., VERDAGUER S. : Valeur prédictive de l'insémination intra-utérine de sperme homologue capacité en cas d'infertilité du couple. Contracept. Fertil. Steril., 1988, 16: 809-815.

10. FRIEDMAN A.J., JUNEAU-NORCROSS M., SEDENSKY B., ANDREWS N., DORFMAN J., CRAMER D.W. : Life table analysis of intrauterine insemination pregnancy rate for couples with cervical factor male factor and idiopathic infertility. Fertil. Steril., 1991, 55: 1005-1007.

11. GALLE P.C., MC RAE M.A., COLLIVER J.A. AND ALEXANDER J.S. : Sperm washing and intrauterine insemination for cervical factor, oligospermia., immunologic infertility and unexplained infertility. J. Reprod. Med., 1990, 35, 116-122.

12. GERRIS J.M., DELBEKE L.O., PLNJABI U. AND BUYTAERT P. : The value of intra-uterine insemination with washed husband's sperm in the treatment of infertility. Hum. Reprod., 1987,2, 315-320.

13. GUERIN J.F., MATHIEU C., LORNAGE J., PINATEL M.C., BOULIEU D. : Improvement of survival and fertilizing capacity of human spermatozoa in an IVF program by selection on discontinuous Percoll gradient. Human . Reprod., 1989, 4: 798-804.

14. HO P.C., POON I.M.L., CHAN S.Y.W. AND WANG C. : Intra-uterine insemination is not useful in oligo asthenospermia. Fertil. Steril., 1989, 51, 682684.

15. HOING L.M., DEVROEY P. AND VAN STEIRTEGHEM A.C. : Treatment of infertility because of oligoasthenospermia by transcervical intra-uterine insemination of motile spermatozoa. Fertil. Steril., 1987, 48, 278-281.

16. KALBFLEISH J.D., PRENTICE R.L. : The statistical analysis of failure time data. Wiley New York, 1980.

17. KERIN J.F.P., PEEK J., WARNES G.M., KIRBY C., JEFFREY R., MATTHEWS C.D., COX L.M. : Improved conception rate after intra uterine ingemination of washed spermatozoa from men with poor quality sperm. Lancet., 1984, 1: 533-535.

18. KERIN J.F.P., QUINN P. : Washed intra-uterine insemination in the treatment of oligospermic infertility. Sem. Reprod, Endocrinol., 1987, 1: 23-33.
19. LALICH R.A., MARUT E.L., PRINS G.S. AND SCOMMEGNA A. : Life table analysis of intrauterine insemination pregnancy rates. Am. J. Obst. Gynecol., 1988,158, 980-984.

20. MATHIEU C., MEIN, M., LORNAGE, J., LI, J.G. AND GUERIN J.F. : Effect of spermatozoa selection on a simplified percoll gradient in case of athenozoospermia. Andrologia., 1990,22, 467 471.

21. SCEMAMA H., SALAT-BAROUX J., ANTOINE J.M., SAADA H. AND COHEN-BACRIE P. : L'hyperstimulation contrôlée du cycle associée à une insémination intra utérine de sperme migré du conjoint. Gynécologie.,1993, 1, 125-131.

22. SCHWARTZ D.: Intérêt du taux actuariel de grossesse dans l'évaluation du traitement de la stérilité. Contracept. Fertil. Sex., 11, 227-233.

23. SCHWARTZ D., MAYAUX M.J., GUILHARDMOSCATO M.L., SPIRA A., JOUANNET P., CZYGLIK F. AND DAVID G. : Semen characteristics as a function of age in 833 fertile men. Fertil. Steril.,1983, 39, 530-535.

24. SCHWARTZ D., MAYAUX M.J. AND DE MOUZON J. : Influence de l'âge, de l'environnement et d'autres facteurs sur la fertilité de la femme. In recherches récentes sur l'épidémiologie de la fertilité. Masson Paris,1986, p 75-80.

25. TAN S.L., ROYSTON P., CAMPBELL S., JACOBS H.S., BETTS J., MASON B. AND EDWARDS R.G. : Cumulative conception and live birth rates after in vitro fertilization. Lancet., 1992, 339, 13901394.

26. TE VELDE E.R., VAN KOOY R.J. AND WATERREUS J.J.H. : Intra-uterine insemination of washed husband's spermatozoa : a controlled study. Fertil. Steril., 1989, 51, 182-185.

27. THEPOT F., WACK T., SELVA J., CZYGLIK F. AND MAYAUX M.S. : Age paternel et issues de grossesses. Expérience r'es CECOS. Contracept. Fertil. Sex., 1993, 21, 388-s.:,

28. THOMPSON W.A. : On the treatment of grouped observations in life studies. Biometrics., 1977, 33, $463-470$.

29. TOFFLE R.C., NAGEL T.C., TAGATZ G.E., PHANSEY S.A., OKAGAKI T. AND WAVRIN C.A. : Intra-uterine insemination: the University of Minnesota experience. Fertil. Steril., 1985, 43, 743747 .

30. TROUNSON A., YATES C., TEMPLE-SMITH P., SOUTHWICK G., LACHAM O. : The values of IVF for male infertility management in Perspective in Andrology. M. Serio ed. SERONO Symposia Publications. Raven New York, 1989, p 420429. 


\section{ABSTRACT}

Uterine Artificial Insemination Homologous in couples with male infertility: role of the husband's age.

C. MAThieu, R. Ecochard, V. BiEd, J. LoRNAGE, J.C. CZYBA

This paper presents the analysis of 492 cycles of Intra Uterine Artificial Insemination Homologous (IU AIH) in 152 couples with male infertility ; 36 pregnancies were achevied. We used the life table method and the Cox model to analyse the results and to determine prognosis factors. Univariate analysis did not disclose any prognostic factor.
Multivariate analysis revealed 3 negative prognostic factors : husband's age $\geq 35$ years ( $p: 0.04$ ), dysovulation, secondary infertility in man. On the contrary, secondary infertility in woman and oligozoospermia were found to be of good prognosis. Surprisingly, the wife's age $\geq 35$ years did not appear to be of poor prognosis in the multivariate analysis. The husband's age has been shown to play a role in fertility by others authors using the Cox method.

Key words : Artificial insemination Male infertility Oligoasthenoteratozoospermia Life table. Cox method. 